\title{
Análise da Relação Custo-Benefício de Projeto de Eficiência Energética no Setor Residencial
}

\author{
Analysis of Cost-Effective Project Energy Efficiency in the Residential Sector \\ Arnulfo Barroso de Vasconcellos, Luciana Oliveira da Silva Lima e Carlos Henrique \\ Beuter, \\ Faculdade de Arquitetura Engenharia e Tecnologia, Universidade Federal de Mato Grosso - UFMT, Cuiabá/MT, Brasil, (arnulfo@ufmt.br). \\ Teresa Irene Ribeiro de Carvalho Malheiro \\ PhD. Instituto Federal de Educação, Ciência e Tecnologia de Mato Grosso - IFMT, Cuiabá/MT, Brasil. \\ Gisele Auxiliadora de Almeida Rios \\ Universidade Estadual Paulista "Júlio de Mesquita Filho" - UNESP Ilha Solteira/SP - Brasil.
}

\begin{abstract}
Recebido em 14/03/2013 aceito em 15/03/2013 Publicado:08/05/2014
RESUMO - A troca de refrigeradores antigos e ineficientes por refrigeradores eficientes Selo Procel classe A e de lâmpadas incandescentes por fluorescente compactas em Unidades Consumidoras de baixa renda provoca, além do aumento da eficiência energética, impacto na vida do consumidor. Entretanto, uma nova modalidade de projetos de eficiência energética em edificações propõe a redução do consumo de energia elétrica e de demanda de potência ativa em função da melhoria de sua envoltória. O uso racional da energia elétrica, ou seja, sem desperdícios, leva ao aumento da confiabilidade do sistema elétrico, reduz ou posterga as necessidades de investimentos em geração, transmissão e distribuição, além dos impactos ambientais (locais e globais), e a simulação computacional tem-se mostrado uma ferramenta eficaz para avaliar projetos de eficiência energética.
\end{abstract}

Palavras-chave: Eficiência energética. Retrofit. Relação Custo-Benefício.

\begin{abstract}
The exchange of old, inefficient refrigerators for efficient refrigerators Procel Class A and incandescent for fluorescent compact Consumer Units in low-income lamps causes, in addition to increased energy efficiency, impact on the consumer. However, a new type of energy efficiency projects in buildings proposes to reduce the electricity consumption and demand of active power due to the improvement of its envelope. The rational use of electricity, ie, without waste, leads to increased reliability of the electric system, reducing or postponing the need for investment in generation, transmission and distribution, and environmental impacts (local and global), and computer simulation has proved an effective tool for evaluating energy efficiency projects.
\end{abstract}

Keywords: Energy Efficiency. Retrofit. Cost Benefit.

\section{INTRODUÇÃO}

O projeto "Luz em Conta", desenvolvido pelas Concessionárias de energia elétrica desde 2004, realiza a troca gratuita de lâmpadas incandescentes de $100 \mathrm{~W}$ por lâmpadas fluorescentes compactas de $20 \mathrm{~W}$ e geladeiras velhas por refrigeradores de $300 \mathrm{~L}$ com Selo Procel, de famílias carentes. Os beneficiados pelo programa entregam $o$ eletrodoméstico velho e as lâmpadas à concessionária, que lhes dá fim ecologicamente adequado.

Desta forma, ocorre a redução no consumo de energia elétrica das unidades consumidoras atendidas pelo projeto, visto que as lâmpadas e refrigeradores substituídos apresentam um consumo elevado de eletricidade, impactando na redução da ponta da curva de carga do sistema elétrico.

$\mathrm{O}$ novo refrigerador permite um melhor acondicionamento dos alimentos, além de diminuir o valor da conta de luz do usuário beneficiado, proporcionando condições para que o mesmo tenha condições de manter-se adimplente.

A implantação do projeto prevê uma redução de $30 \%$ a $60 \%$ nas faturas de energia elétrica dos usuários atingidos pelo projeto. Outro objetivo secundário é a redução da 
ponta na curva de carga do consumidor e consequente reflexo na Curva de Carga do Sistema.

Porém, é sentida uma grande dificuldade por parte das concessionárias para se estimar o consumo e a demanda do sistema convencional e ineficiente, ante a variedade de situações encontradas em campo e o elevado custo da medição de casos específicos em projetos desta natureza, o que torna a simulação computacional vantajosa.

A simulação computacional tem se mostrado uma poderosa ferramenta que permite analisar a redução do consumo de energia elétrica e o desempenho termo energético da edificação. Para as simulações utilizou-se o software Energyplus que é um programa computacional, criado a partir dos programas BLAST e DOE-2, distribuído gratuitamente pelo Departamento de Energia dos Estados Unidos2. Foi desenvolvido para simulação de carga térmica, consumo de energia elétrica ativa, estimativa de demanda de potência ativa, enquadramento tarifário mais adequado e análise energética de edificações e seus sistemas. Por meio do Plug-in OpenStudio, o SketchUp, programa de criação de maquetes eletrônicas em 3D, é integrado ao Energyplus, o que possibilita a edição e visualização em 3D da edificação simulada.

\section{ÁREA DE ESTUDO}

O estudo foi realizado para a substituição de 2.400 refrigeradores e de 9.600 lâmpadas de $100 \mathrm{~W}$, sendo adotada uma casa popular tipo, localizada na cidade de Cuiabá-MT. Cuiabá possui dois períodos bem definidos: um quente e seco (abril-outubro) e outro quente e úmido (novembro-março). Dispõe de pequena amplitude térmica, com uma temperatura média anual de $26,8^{\circ} \mathrm{C}$.

Embora o projeto de troca lâmpadas e refrigeradores sejam executados em várias cidades de Mato Grosso, verifica-se a ocorrência de temperaturas elevadas na capital do Estado, o que exige mais dos sistemas avaliados. Desta forma, foi utilizado o arquivo climático de Cuiabá para as simulações computacionais.

A casa escolhida possui sala e cozinha conjugadas, dois dormitórios e um banheiro, com paredes em alvenaria e cobertura em telha de cerâmicas com forro em PVC. Este modelo foi escolhido em virtude de ser o tipo utilizado para receber famílias carentes removidas de áreas de risco da capital. $\mathrm{Na}$ Figura 1 é apresentada a planta baixa da edificação tipo e os detalhes construtivos das paredes e da cobertura.

Figura 1 - Planta Baixa da edificação tipo e detalhe construtivo da parede e da cobertura

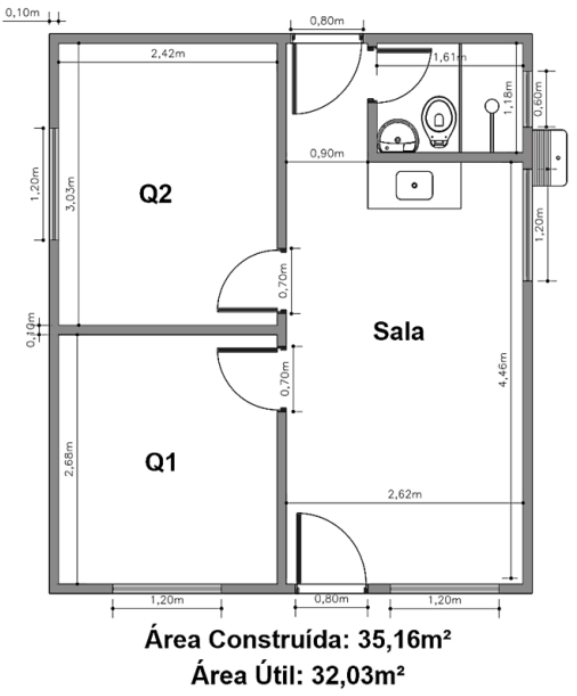

Fonte: Adaptado de [4]

As características construtivas da envoltória e da cobertura da edificação são importantes, pois interferem no consumo energético da edificação.

\section{Modelagem da Casa Popular}

A simulação da casa popular passou por diversas etapas, incluindo sua modelagem no software Google SketchUp, onde por meio do plug-in OpenStudio, foi feita a conexão com o EnergyPlus. Para a modelagem, a casa foi dividida em zonas térmicas. As características geométricas e as propriedades térmicas dos elementos construtivos da edificação real foram preservadas para cada zona térmica. $\mathrm{O}$ beiral do telhado foi modelado como objeto de sombreamento. A Figura 2 apresenta a divisão das zonas 
Citação: Vasconcellos A. B., et al Análise da Relação Custo-Benefício de Projeto de Eficiência Energética no Setor Residencial. E\&S Engineering and Science, (2014), 1:1

térmicas e a maquete eletrônica 3D da casa popular analisada.

Figura 2 - Divisão das zonas térmicas da casa popular tipo e Maquete eletrônica 3D da edificação.

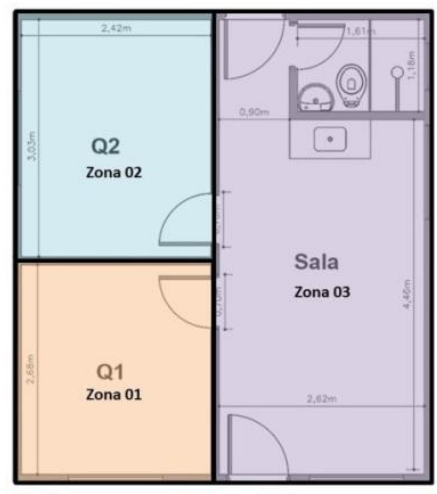

Após a modelagem geométrica da casa popular, foi gerado um arquivo com a extensão. idf, contendo as informações da superfícies modeladas da edificação. Para realizar a simulação, é necessário fornecer ao EnergyPlus a temperatura do solo média mensal da região onde está localizada a

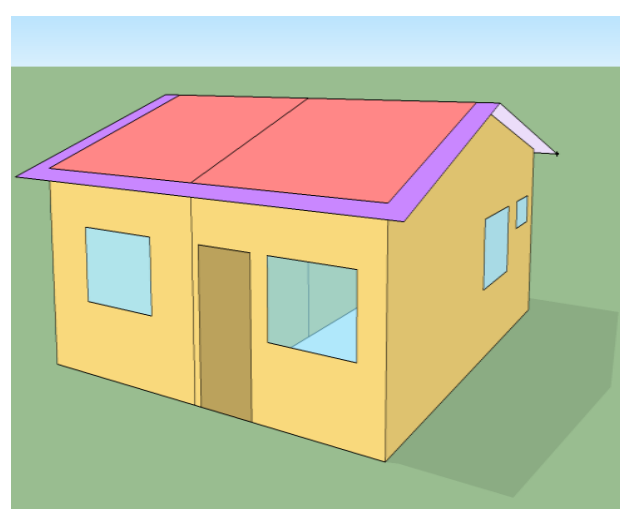

edificação. Para o cálculo destas temperaturas, o EnergyPlus recomenda a utilização de um programa auxiliar, chamado Slab. A Tabela 1 apresenta as médias mensais da temperatura do solo, obtidas utilizando o arquivo modelo do Slab com o arquivo climático da cidade de Cuiabá.

\begin{tabular}{llll}
\hline \multicolumn{1}{c}{ Mês } & Temperatura Média $\left({ }^{\circ} \mathrm{C}\right)$ & Mês & Temperatura Média $\left({ }^{\circ} \mathrm{C}\right)$ \\
\hline Janeiro & 18,05 & Julho & 21,12 \\
Fevereiro & 17,79 & Agosto & 21,36 \\
Março & 17,77 & Setembro & 21,39 \\
Abril & 19,29 & Outubro & 21,42 \\
Maio & 19,54 & Novembro & 19,91 \\
Junho & 19,56 & Dezembro & 19,65 \\
\hline
\end{tabular}

Tabela 1 - Médias Mensais da Temperatura do Solo utilizadas na simulação.

Também foram cadastrados no edificação, com suas propriedades térmicas, EnergyPlus os materiais construtivos da conforme a Tabela 2.

\begin{tabular}{llllcc}
\hline Material & $\begin{array}{l}\text { Densidade de massa } \\
\text { aparente }(\rho)\left(\mathrm{kg} / \mathrm{m}^{3}\right)\end{array}$ & $\begin{array}{l}\text { Cond. Térmica }(\lambda) \\
(\mathrm{W} / \mathrm{mK})\end{array}$ & $\begin{array}{l}\text { Calor Específico }(\mathrm{c}) \\
(\mathrm{J} / \mathrm{kgK})\end{array}$ & $\begin{array}{l}\text { Abs. Solar }(\alpha) \\
\text { Abs. Térmica }(\varepsilon)\end{array}$ \\
\hline Argamassa & 2100 & 1,15 & 1000 & 0,97 & 0,9 \\
Concreto & 1400 & 1,75 & 1000 & - & - \\
Forro PVC & 1400 & 0,19 & - & - & 0,8 \\
Telha Cerâmica & 1600 & 0,9 & 920 & 0,8 & 0,95 \\
Tijolo Cerâmico & 1600 & 0,9 & 920 & 0,8 & 0,95 \\
\hline
\end{tabular}

Tabela 2 - Características térmicas dos materiais da edificação tipo

Os valores médios das características de cada sistema simulado inseridas no Modelo
Base de simulação estão sintetizados na Tabela 3 . 
Citação: Vasconcellos A. B., et al Análise da Relação Custo-Benefício de Projeto de Eficiência Energética no Setor Residencial. E\&S Engineering and Science, (2014), 1:1

\begin{tabular}{lllll}
\hline Variável & $\mathrm{SR}_{\mathrm{C}}$ & $\mathrm{SR}_{\mathrm{E}}$ & $\mathrm{SI}_{\mathrm{C}}$ & $\mathrm{SI}_{\mathrm{E}}$ \\
\hline Quantidade & 01 & 01 & 04 & 04 \\
Potência Unitária (W) & 112 & 94 & 100 & 22 \\
Potência Total (W) & 112 & 94 & 400 & 88 \\
Tempo diário de funcionamento (h) & 17 & 09 & 03 & 03 \\
\cline { 2 - 3 }
\end{tabular}

Tabela 3 - Padrões de simulação

Onde:

- $\quad \mathrm{SR}_{\mathrm{C}}$ : Sistema de Refrigeração Convencional;

- $\mathrm{SR}_{\mathrm{E}}$ : Sistema de Refrigeração Eficiente;

- $\mathrm{SI}_{\mathrm{C}}$ : Sistema de Iluminação Convencional;

- $\mathrm{SI}_{\mathrm{E}}$ : Sistema de Iluminação Eficiente;

Figura 3 - Consumo e Demanda dos sistemas de Refrigeração e de Iluminação, respectivamente.
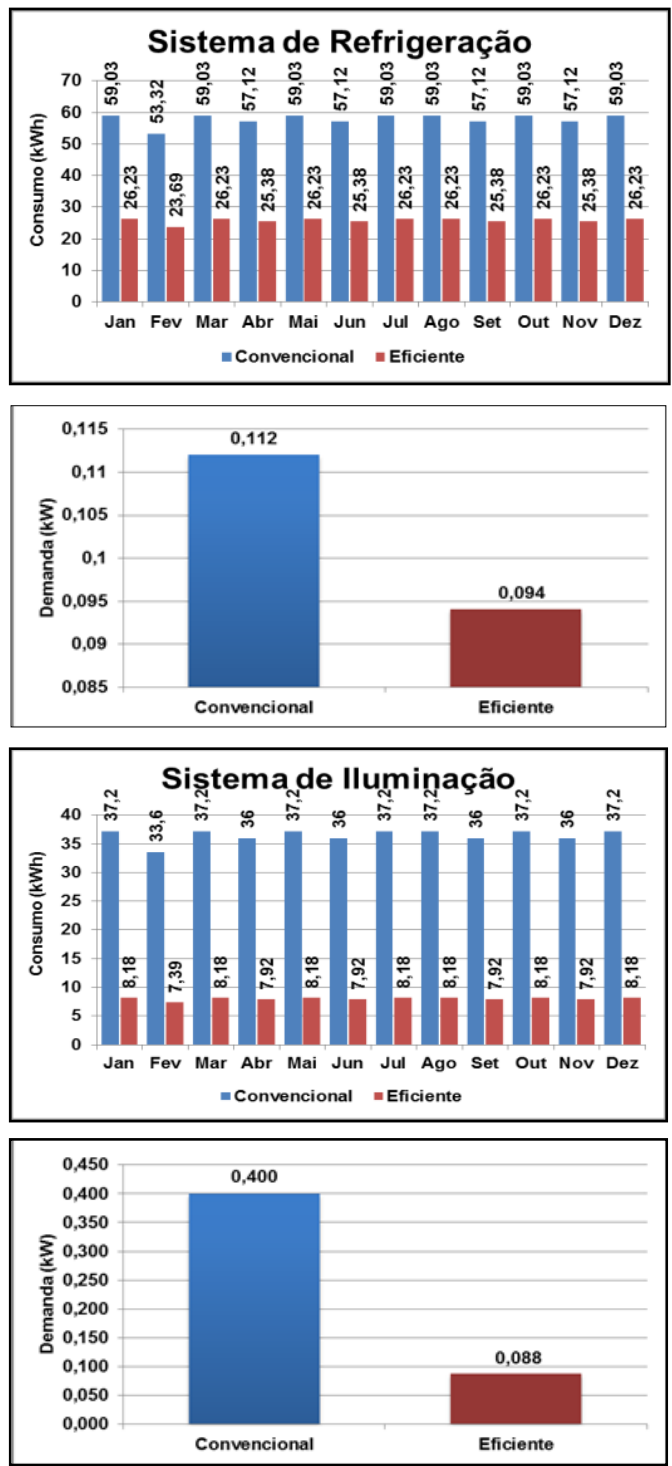

O tempo de funcionamento do refrigerador eficiente é menor, em função da melhor vedação da borracha da porta, o que leva a um menor tempo de funcionamento do compressor.

\section{Simulação Computacional no Software EnergyPlus.}

Após a parametrização de cada sistema, foi executada a simulação computacional, sendo solicitado no relatório de saída, o consumo mensal e a demanda da edificação tipo, apresentados na Figura 3.

Devido à adoção de valores médios para a Potência Unitária e Tempo de Funcionamento na casa tipo, e como em uma distribuição normal de dados, tem-se uma concentração maior de probabilidades de ocorrência de dados em torno da média, supõem-se que a estimativa do consumo de energia elétrica e de demanda seja semelhante para as 2.400 unidades consumidoras. Com uma taxa de variação aceitável de $\pm 5 \%$ em torno da média, foram criada em uma planilha eletrônica, a expansão da estimativa do consumo anual de energia elétrica para cada sistema avaliado, totalizados na Tabela 4.

\begin{tabular}{|c|c|c|c|c|}
\hline $\begin{array}{l}2.400 \\
\text { residências }\end{array}$ & $\mathrm{SR}_{\text {Convencional }}$ & $\mathrm{SR}_{\text {Eficiente }}$ & $\mathrm{SI}_{\text {Convencional }}$ & SI $\mathrm{I}_{\text {Eficiente }}$ \\
\hline $\begin{array}{c}\text { Consumo } \\
\text { Anual Total } \\
(\mathrm{kWh} / \mathrm{ano})\end{array}$ & $\begin{array}{c}1.669 .518,0 \\
0\end{array}$ & $741.797,30$ & $\begin{array}{c}1.052 .223,0 \\
0\end{array}$ & $231.491,90$ \\
\hline $\begin{array}{c}\text { Potência } \\
\text { Total }(\mathrm{kW})\end{array}$ & 269,03 & 225,80 & 960,84 & 211,39 \\
\hline
\end{tabular}

Tabela 4 - Estimativa do Consumo Anual de energia elétrica e da Potência Total

As estimativas de consumo anual de energia elétrica e a potencia total serão utilizados no cálculo da relação custobenefício. 
Citação: Vasconcellos A. B., et al Análise da Relação Custo-Benefício de Projeto de Eficiência Energética no Setor Residencial. E\&S -

Cálculo da Energia Economizada (EE) e da Redução de Demanda na Ponta (RDP)

A Energia Economizada (EE), em MWh, e a Redução de Demanda na Ponta (RDP), em KW, são os indicadores quantitativos mais importantes para programas de eficiência energética. As economias de energia ou reduções de demanda são determinadas através da comparação dos usos estimados de energia ou demanda para o sistema convencional e eficiente. Desta forma, o Manual Para Elaboração do Programa de Eficiência Energética estabelece a metodologia para o calculo da RDP e EE para cada sistema avaliado.

\section{Sistema de Iluminação}

A RDP do Sistema de Iluminação e a EE são dadas pelas equações um e dois, respectivamente.

$$
\begin{aligned}
& R D P_{\text {Iluninaçăo }}=\left[\left(N L_{1} \times P L_{1}\right)-\left(N L_{2} \times P L_{2}\right)\right] \times F C P \times 10^{-3}(k W) \\
& E E_{\text {lluminaç̃õo }}=\left[\left(N L_{1} \times P L_{1}\right)-\left(N L_{2} \times P L_{2}\right)\right] \times t \times 10^{-6}(M W h)
\end{aligned}
$$

Onde:

$\mathrm{NL}_{1}$ : Quantidade de lâmpadas do sistema existente

$\mathrm{NL}_{2}$ : Quantidade de lâmpadas do sistema proposto

$\mathrm{PL}_{1}$ : Potência da lâmpada do sistema existente (W)

$\mathrm{PL}_{2}$ : Potência da lâmpada do sistema proposto $(\mathrm{W})$

t: Tempo de utilização das lâmpadas no ano, em horas $=3 \mathrm{~h} /$ dia $\times 365$ dias $/$ ano $=1095$

FCP: Fator de coincidência na Ponta: 0,70

Entretanto, o termo $\left(N L_{1} \times \quad P L_{1}\right)$ das Equações 2 e 3 pode ser substituído pela Potência Total do $\mathrm{SI}_{\text {convencional }}$ da Tabela 4, enquanto o termo $\left(N L_{2} \times P L_{2}\right)$ pode ser substituído pela Potência Total do $\mathrm{SI}_{\text {eficiente, }}$ da referida Tabela. Desta forma, têm-se:

$$
\begin{aligned}
& \left(N L_{1} \times P L_{1}\right)=\text { PotênciaTotal }(\mathrm{kW}) S I_{\text {Convencional }}=960,84(\mathrm{~kW}) \\
& \left(N L_{2} \times P L_{2}\right)=\text { PotênciaTotal }(k W) S I_{\text {Efficiente }}=211,39(\mathrm{~kW}) \\
& R D P_{\text {llu min a as̃o }}=[960,84-211,39] \times 0,7(\mathrm{~kW})
\end{aligned}
$$

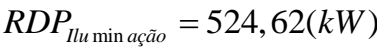

$$
\begin{aligned}
& \begin{array}{l}
E E_{\text {Ilu min ação }}=[960,84-211,39] \times 1.095 \times 10^{-3}(M W h) \\
E E_{\text {Ilu min ação }}=820,65(M W h)
\end{array}
\end{aligned}
$$

Para o Sistema de Iluminação, obteve-se uma RDP de 524,62KW e uma EE de 820,62MWh.

\section{Sistema de Refrigeração}

A EE do Sistema de Refrigeração e a RDP são dadas pelas Equações 3 e 4, respectivamente.

$$
E E_{\text {Refrigerặão }}=N \times\left(C_{1}-C_{2}\right) \times 10^{-3}(M W h / \text { ano })
$$

$$
R D P_{\text {Refrigeração }}=\frac{E E_{\text {Refrigeração }} \times F U \times 10^{3}}{t}(\mathrm{~kW})
$$

Onde:

$\mathrm{C}_{1}$ : Consumo de energia dos equipamentos convencionais;

$\mathrm{C}_{2}$ : Consumo de energia dos equipamentos eficientes;

$\mathrm{N}: \mathrm{n}^{\circ}$ de refrigeradores.

FU: Fator de utilização médio $=1,25$ (devido á utilização do refrigerador, abertura e fechamento de porta);

t: Tempo de utilização do equipamento de refrigeração no ano horas $=8.760 \mathrm{~h}$;

Entretanto, o termo $N \times\left(C_{1}-C_{2}\right)$ da Equação 3 é a diferença entre o Consumo Anual Total o $\mathrm{SR}_{\text {convencional }}$ e o $\mathrm{Ef}_{\text {iciente }} \mathrm{da}$ Tabela 4. Logo, têm-se: 
Citação: Vasconcellos A. B., et al Análise da Relação Custo-Benefício de Projeto de Eficiência Energética no Setor Residencial. E\&S Engineering and Science, (2014), 1:1

$$
\begin{aligned}
& N \times\left(C_{1}-C_{2}\right)=1.669 .518,00-741.797,30(\mathrm{kWh} / \text { ano }) \text { do que o obtido se o recurso fosse } \\
& N \times\left(C_{1}-C_{2}\right)=927.720,70(\mathrm{kWh} / \text { ano }) \\
& E E_{\text {Re frigerasăo }}=927,721(M W h / \text { ano }) \\
& R D P_{\text {Re frigeração }}=\frac{927,721 \times 1,25 \times 10^{3}}{8760}(\mathrm{~kW}) \\
& R D P_{\text {Re frigeraça o o }}=132,38(\mathrm{~kW})
\end{aligned}
$$

A Energia Economizada Total $\left(\mathrm{EE}_{\text {Total }}\right)$ e a Redução de Demanda na Ponta Total $\left(\mathrm{RDP}_{\text {Total }}\right)$ são dadas pela soma da $\mathrm{EE}$ e da RDP de cada sistema. Sendo Assim, têm-se:

$$
\begin{aligned}
& E E_{\text {Total }}=E E_{\text {Ilu min ą̃ão }}+E E_{\text {Re frigeração }}(M W h / \text { ano }) \\
& E E_{\text {Total }}=820,65+927,72 \\
& E E_{\text {Total }}=1.748,37(M W h / \text { ano }) \\
& R D P_{\text {Total }}=R D P_{\text {Iluminação }}+R D P_{\text {Re frigeração }}(k W) \\
& R D P_{\text {Total }}=524,62+132,38(\mathrm{~kW}) \\
& R D P_{\text {Total }}=657,00(\mathrm{~kW})
\end{aligned}
$$

De posse da estimativa de redução do consumo anual de energia elétrica e de demanda na ponta, é possível calcular a relação custo-benefício.

\section{Viabilidade Econômica de Projetos de EFICIÊNCIA ENERGÉTICA}

Com o objetivo de orientar o cálculo da viabilidade econômica de Projetos de Eficiência Energética (PEE), a ANEEL estabeleceu através da Resolução Normativa $\mathrm{n}^{\circ} 556 / 2013$ de 02 de Julho de 2013 os Procedimentos do Programa de Eficiência Energética - PROPEE, que no Módulo 7, trata do Cálculo da Viabilidade dos Projetos.

O parâmetro fundamental utilizado para avaliação da viabilidade econômica de um projeto do PEE é a Relação Custo-Benefício (RCB) que ele assegura. $\mathrm{O}$ benefício considerado é a valoração da energia economizada e da redução da demanda na ponta durante a vida útil do projeto para o sistema elétrico. O custo são os aportes feitos para a sua realização (do PEE, do consumidor ou de terceiros).

A racionalidade da avaliação de um projeto de eficiência energética está em saber se o benefício proporcionado é maior

Do ponto de vista do sistema elétrico, a economia de energia e a redução de demanda podem ser valoradas pela tarifa azul, ou tarifa do sistema de bandeiras tarifárias de energia, segundo estabelecido no Módulo 7 dos Procedimentos de Regulação Tarifária (PRORET).

\section{Custo Evitado de Demanda (CED)}

O Custo Evitado de Demanda (CED) unitário para projetos de eficiência em Baixa Tensão é valorado na tarifa azul, sendo calculado através da Equação 5.

$$
C E D=\left(12 \times C_{1}\right)+\left(12 \times C_{2} \times L P\right)
$$

Onde:

12: meses (mês/ano);

$\mathrm{C}_{1}$ : Custo unitário da demanda no horário de ponta (R\$/kW.mês);

$\mathrm{C}_{2}$ : Custo unitário da demanda no horário fora de ponta (R\$/kW.mês);

LP: Constante de perda de demanda no posto fora de ponta.

\section{Custo da Energia Evitada (CEE)}

O Custo da Energia Evitada (CEE) unitário é realizado por meio da Equação 6, para concessionárias que possuem o sistema de bandeiras tarifárias, na tarifa azul, e as Equações 7 e 8 representam as constantes de perda de energia no horário de ponta e fora de ponta, respectivamente.

$$
C E E=\frac{\left(C_{p} \times L E_{p}\right)+\left(C_{f p} \times L E_{f p}\right)}{L E_{p}+L E_{f p}}
$$


Citação: Vasconcellos A. B., et al Análise da Relação Custo-Benefício de Projeto de Eficiência Energética no Setor Residencial. E\&S Engineering and Science, (2014), 1:1

$$
\begin{aligned}
& L E_{p}=\frac{\left(7 \times L E_{1}\right)+\left(5 \times L E_{2}\right)}{12} \\
& L E_{f p}=\frac{\left(7 \times L E_{3}\right)+\left(5 \times L E_{4}\right)}{12}
\end{aligned}
$$

Onde:

$\mathrm{C}_{\mathrm{p}}$ : Custo unitário da energia no horário de ponta na bandeira verde ( $\mathrm{R} \$ \mathrm{MWh})$;

$\mathrm{C}_{\mathrm{fp}}$ : Custo unitário da energia no horário fora de ponta na bandeira verde ( $\mathrm{R} / \mathrm{MWh})$;

$\mathrm{LE}_{\mathrm{p}}$ : Constante de perda de energia no posto de ponta;

$\mathrm{LE}_{\mathrm{fp}}$ : Constante de perda de energia no posto de fora de ponta;

$\mathrm{LE}_{1}$ : Constante de perda de energia no posto de ponta de períodos secos;

$\mathrm{LE}_{2}$ : Constante de perda de energia no posto de ponta de períodos úmidos;

$\mathrm{LE}_{3}$ : Constante de perda de energia no posto fora de ponta de períodos secos;

$\mathrm{LE}_{4}$ : Constante de perda de energia no posto fora de ponta de períodos úmidos.

Este método se baseia no cálculo do custo unitário de perdas técnicas no sistema elétrico, onde a economia de energia e demanda correspondem a uma redução de perdas no sistema e o benefício "de evitar uma unidade de perdas é numericamente igual ao custo de fornecer uma unidade adicional de carga".

O cálculo se baseia no impacto para o sistema da carga evitada, supondo-se um perfil de carga típico e caracterizado pelo Fator de Carga (Fc). As perdas evitadas no sistema são calculadas a partir da redução de $1 \mathrm{~kW}$ na ponta, seu reflexo na demanda fora de ponta (LP) através do fator de carga, e pelos fatores de perda $(\mathrm{Fp})$, que levam ao cálculo de LE1, LE2, LE3 e LE4, juntamente com a permanência de cada posto horário no ano - 450, 315, 4.686 e 3.309 h/ano respectivamente), que medem o reflexo desta redução no horário fora de ponta e na energia consumida nos 4 postos tarifários (seco e úmido, ponta e fora de ponta). $\mathrm{O}$ fator de perda pode ser simulado através do fator de carga pela expressão 9.

$$
\mathrm{Fp}=\mathrm{k} \times \mathrm{Fc}+(1-\mathrm{k}) \times \mathrm{Fc}^{2}
$$

Onde:

$\mathrm{k}$ varia tipicamente de 0,15 a 0,30 . Recomenda-se adotar $\mathrm{k}=0,15$;

Fc: Fator de carga médio da distribuidora dos últimos 12 meses.

Na Tabela 5 são apresentados os coeficientes das Equações 7, 8 e 9.

\begin{tabular}{cccccc}
\hline Fc & LP & LE1 & LE2 & LE3 & LE4 \\
\hline 0,55 & 0,4225 & 0,33750 & 0,23625 & 1,40748 & 0,99389 \\
0,60 & 0,4624 & 0,35199 & 0,24639 & 1,68249 & 1,18808 \\
0,65 & 0,5041 & 0,36950 & 0,25865 & 1,97632 & 1,39557 \\
\hline
\end{tabular}

Tabela 5 - Coeficientes das Equações 7,8 e 9 $(\mathrm{k}=0,15)$

\section{Relação Custo-Benefício (RCB)}

A avaliação econômica do projeto é feita por meio do cálculo da relação CustoBenefício (RCB) para cada uso final. Assim, os benefícios são avaliados como a soma das energias conservadas, em uma base anual, decorrente da diminuição de perdas pela geração descentralizada mais aquela devido à substituição de equipamentos elétricos valorizados pelos custos marginais, obedecendo à metodologia seguinte, onde equipamentos significam lâmpadas e refrigeradores. Logo, a RCB é dada pela Equação 10.

$$
R C B=\frac{C A_{T}}{B A_{T}}
$$

Onde:

$\mathrm{CA}_{\mathrm{T}}$ : Custo anualizado total (R $\left.\$ / \mathrm{ano}\right)$

$\mathrm{BA}_{\mathrm{T}}$ : Benefício anualizado (R $\left.\$ / \mathrm{ano}\right)$ 
Citação: Vasconcellos A. B., et al Análise da Relação Custo-Benefício de Projeto de Eficiência Energética no Setor Residencial. E\&S -

Custo Anualizado dos Equipamentos

$\left(\mathbf{C A}_{\mathbf{n}}\right)$

$\mathrm{O} \mathrm{CA}_{n}$ é obtido através do produto do custo de cada equipamento, pela relação do Custo Total em Equipamentos e pelo Fator de Recuperação de Capital (FRC), segundo as Equações 11 e 12.

$$
\begin{aligned}
& C A_{n}=C E_{n} \times \frac{C T}{C E_{T}} \times F R C_{u} \\
& F R C_{u}=\frac{i(1+i)^{u}}{(1+i)^{u}-1}
\end{aligned}
$$

Onde:

$\mathrm{CE}_{\mathrm{n}}$ : Custo de cada equipamento ( $\left.\mathrm{R} \$\right)$;

$\mathrm{CT}$ : Custo total do projeto ( $\mathrm{R} \$)$;

$\mathrm{CE}_{\mathrm{T}}$ : Custo total em equipamentos $(\mathrm{R} \$)$;

u: Vida útil dos equipamentos, em anos;

i: Taxa de desconto.

O FRC é um importante valor de referência para análise de retorno de investimentos e amortização de capital (empréstimos e financiamentos). Este fator está relacionado a estimativa do valor da parcela a ser debitada na fatura de energia elétrica da Unidade Consumidora beneficiada pela implantação do Projeto de Eficiência Energética em função da vida útil dos equipamentos utilizados.

A taxa de desconto considerada é a mesma especificada no Plano Nacional de Energia (i=8\%) e a vida útil dos equipamentos é adotada com base nos dados fornecidos pelo fabricante de cada equipamento.

\section{Benefícios Anualizados $\left(\mathbf{B} \mathbf{A}_{\mathrm{T}}\right)$}

$\mathrm{O} \mathrm{BA}_{\mathrm{T}}$ é a valoração dos benefícios de economia de energia elétrica e de redução de demanda na ponta, sendo obtida através da Equação 15.

$$
B A_{T}=(E E \times C E E)+(R D P \times C E D)
$$

Onde:

$\mathrm{BA}_{\mathrm{T}}$ : Benefício anualizado (R\$/ano)

EE: Energia anual economizada (MWh/ano);

CEE: Custo unitário da energia (R\$/MWh);

RDP: Demanda evitada na ponta $(\mathrm{kW}$ ano);

CED: Custo unitário evitado da demanda ( $\mathrm{R} \$ / \mathrm{kW}$ ano);

\section{Cálculo da Relação Custo-Benefício (RCB)}

O cálculo da RCB foi realizado segundo a metodologia anteriormente exposta. Para tanto, é preciso obter a Vida Útil $\left(\mathrm{U}_{\mathrm{n}}\right)$, em anos, das lâmpadas e dos refrigeradores, segundo dados fornecidos pelo fabricante de cada produto. Desta forma, têm-se:

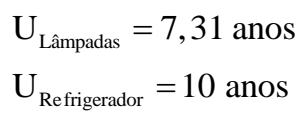

\section{RESULTADOS ESPERADOS}

Como as metas de economia de energia e de demanda podem ser obtidas através de estimativa, será empregada a redução de consumo e de demanda estimados através da simulação computacional, calculados no item 2.3, e sintetizados na Tabela 6.

\begin{tabular}{ccc}
\hline Sistema & RDP $(\mathrm{kW})$ & EE (MWh/ano) \\
\hline Iluminação & 749,45 & 820,731 \\
Refrigeração & 43,23 & 927,721 \\
\hline
\end{tabular}

Tabela 6 - Metas de Redução de Demanda na Ponta (RDP) e de Energia Anual Economizada (EE)

Os Resultados Esperados serão utilizados para valorar a economia de energia e redução de demanda na ponta.

\section{Cálculo do Custo Evitado de Demanda (CED)}

O Custo Evitado de Demanda (CED) foi realizado por meio da Equação 4. C1 e C2 são valores do custo unitário da demanda no horário de ponta e fora do horário de ponta, 
Citação: Vasconcellos A. B., et al Análise da Relação Custo-Benefício de Projeto de Eficiência Energética no Setor Residencial. E\&S -

na tarifa Azul, que valem $\mathrm{R} \$ 30,35$ e $\mathrm{R} \$$ 9,83 por $\mathrm{kW}$, respectivamente.

Porém, para a obtenção da constante de perda (LP) é preciso conhecer o Fator de Carga da concessionária de energia elétrica da região abrangida pelo projeto de eficiência energética.

Para o calculo do Fator de Carga (FC), foi necessário utilizar uma amostragem de dados de consumo de energia elétrica ativa e demanda de potência ativa da concessionária, entre os anos de 2011 e 2012.

O Fator de Carga (FC) é um índice que demonstra se a energia consumida está sendo utilizada de maneira racional e econômica. Este índice varia entre zero a um, e é obtido pela relação entre a demanda média e a demanda máxima, durante um período definido, segundo a Equação 14.

$$
\mathrm{FC}=\frac{\text { Consumo }}{\text { Demanda } \times \text { tempo }}
$$

Desta forma, o FC foi calculado para 730h mensais, apresentado na Tabela 7 , segundo que apresenta a média anual do consumo de energia elétrica registrado pela concessionária de energia elétrica para o ano de 2012, e a máxima demanda registrada neste período.

\begin{tabular}{cccc}
\hline $\begin{array}{c}\text { Meses do } \\
\text { ano }\end{array}$ & $\begin{array}{c}\text { Consumo Energia } \\
(\mathrm{kWh})\end{array}$ & $\begin{array}{c}\text { Dem. Coincid. } \\
(\mathrm{kW})\end{array}$ & FC \\
\hline Média & 454.963 .282 & 1.083 .661 & 0,575 \\
\hline
\end{tabular}

Tabela 7 - Amostragem do Consumo, Demanda e Fator de Carga

O Fator de Carga médio da concessionária foi de 0,575 , sendo adotado um FC de 0,60 para obtenção das Constantes de Perda de Demanda (LP) e Energia (LE) da Tabela 5. Logo, têm-se:

$$
\begin{aligned}
& C E D=(12 \times 30,35)+(12 \times 9,83 \times 0,4624) \\
& C E D=(364,20)+(54,54) \\
& C E D=418,74(R \$ / k W)
\end{aligned}
$$

\section{Cálculo do Custo da Energia Evitada (CEE)}

O CEE foi realizado através das Equações 6, 7 e 8. Segundo o sistema de bandeiras vigente, têm-se:

$$
\begin{aligned}
& C_{p}: 303,18(R \$ / M W h) \text { e } C_{f p}: 192,11(R \$ / M W h) \\
& L E_{p}=\frac{(7 \times 0,35199)+(5 \times 0,24639)}{12} \\
& L E_{p}=0,30799
\end{aligned}
$$

$$
\begin{aligned}
& L E_{f p}=\frac{(7 \times 1,68249)+(5 \times 1,18808)}{12} \\
& L E_{f p}=1,47649 \\
& C E E=\frac{(303,18 \times 0,30799)+(192,11 \times 1,47649)}{0,30799+1,47649} \\
& C E E=211,28(R \$ / M W h)
\end{aligned}
$$

\section{Cálculo dos Custos Anualizados $\left(\mathrm{CA}_{\mathrm{T}}\right)$}

Para a execução de um projeto desta natureza, a Aneel exige que todo o projeto seja orçado. A Tabela 8 apresenta os custos do projeto, com a aquisição de lâmpadas e geladeiras.

\begin{tabular}{ccccc}
\hline Descrição & Unid. & Qtde. & $\begin{array}{c}\text { Valor } \\
\text { Unit. }(\mathrm{R} \$)\end{array}$ & $\begin{array}{c}\text { Valor Total } \\
(\mathrm{R} \$)\end{array}$ \\
\hline $\begin{array}{l}\text { Lâmpada flúor. } \\
\text { compacta 20W }\end{array}$ & pç & 9.600 & 6,00 & $57.600,00$ \\
$\begin{array}{l}\text { Refrigerador } \\
\text { Selo Procel } \\
300 \text { litros }\end{array}$ & pç & 2.400 & 650,00 & $1.560 .000,00$ \\
\multicolumn{2}{c}{ Custo Total dos Equipamentos $(\mathrm{R} \$):$} & $1.617 .600,00$ \\
\hline
\end{tabular}

Tabela 8 - Custos dos Equipamentos

O restante dos custos do projeto, apresentados na Tabela 10, foram calculados em função do percentual dos mesmos em relação aos custos dos equipamentos do projeto executado pela concessionária no ano de 2009, apresentados na Tabela 9. 
Citação: Vasconcellos A. B., et al Análise da Relação Custo-Benefício de Projeto de Eficiência Energética no Setor Residencial. E\&S Engineering and Science, (2014), 1:1

\begin{tabular}{ccc}
\hline Tipo de Custo & $\begin{array}{c}\% \text { do } \\
\text { Custo } \\
\text { Total dos } \\
\text { Materiais }\end{array}$ & $\begin{array}{c}\text { Valor Total } \\
\text { (R\$) }\end{array}$ \\
\hline Compra de & $100 \%$ & $1.617 .600,00$ \\
Materiais/Equipamentos & $7 \%$ & $113.232,00$ \\
Mão de Obra de Terceiros & $1 \%$ & $16.176,00$ \\
Mão de Obra de Própria & $6 \%$ & $97.056,00$ \\
Transporte & $3 \%$ & $48.528,00$ \\
Administração Própria & $3 \%$ & $48.528,00$ \\
Marketing & $13 \%$ & $210.288,00$ \\
Descarte de Materiais & $3 \%$ & $48.528,00$ \\
Medição e Verificação & & $2.199 .936,00$ \\
Custos Totais (CT): & $1 \%$ & $16.028,54$ \\
Auditoria Contábil e Financeira & & $2.215 .964,54$ \\
Total Geral: & & \\
\hline
\end{tabular}

Tabela 9 - Detalhamento dos custos do projeto de eficiência energética.

Desta forma, o cálculo dos Custos Anualizados foi realizado para cada sistema.

a) Sistema de Iluminação

$$
\begin{aligned}
& F R C_{7,31}=\frac{0,08(1+0,08)^{7,31}}{(1+0,08)^{7,31}-1} \\
& F R C_{7,31}=0,18601 \\
& C A_{\text {Lampadas }}=57.600,00 \times \frac{2.199 .936,00}{1.617 .600,00} \times 0,18601 \\
& C A_{\text {Lampadas }}=14.571,28(R \$)
\end{aligned}
$$

b) Sistema de Refrigeração

$$
\begin{aligned}
& F R C_{10}=\frac{0,08(1+0,08)^{10}}{(1+0,08)^{10}-1} \\
& F R C_{10}=0,14903 \\
& C A_{\text {Re frigeradores }}=1.560 .000,00 \times \frac{2.199 .936,00}{1.617 .600,00} \times 0,14903 \\
& C A_{\text {Re frigeradores }}=316.182,05(R \$) \\
& C A_{T}=C A_{\text {Lampadas }}+C A_{\text {Re frigeradores }} \\
& C A_{T}=14.571,28+316.182 \\
& C A_{T}=330.753,33(R \$)
\end{aligned}
$$

\section{Cálculo dos Benefícios Anualizados $\left(B A_{T}\right)$}

$$
\begin{aligned}
& B A_{E}=1.748,371(M W h / \text { ano }) \times 211,28(R \$ / M W h) \\
& B A_{E}=369.395,83(R \$) \\
& B A_{D}=657,00(k W) \times 418,74(R \$ / k W) \\
& B A_{D}=275.112,18(R \$) \\
& B A_{T}=B A_{E}+B A_{D} \\
& B A_{T}=369.395,83(R \$)+275.112,18(R \$) \\
& B A_{T}=644.508,01(R \$)
\end{aligned}
$$

\section{Cálculo da RCB Total}

$$
\begin{aligned}
R C B & =\frac{330.753,33(R \$)}{644.508,01(R \$)} \\
R C B & =0,51
\end{aligned}
$$

Avaliando o RCB do projeto de eficiência energética, que envolve a troca de 2.400 refrigeradores convencionais e ineficientes e de 9.600 lâmpadas incandescentes em 2.400 residências é bem inferior ao limite estabelecido pela ANEEL, que considera uma RCB viável para projetos de eficiência energética desta natureza, os valores abaixo de 0,8 , segundo as equações anteriormente expostas.

\section{CONCLUSÕES}

Os custos com energia elétrica representam hoje, para todos os consumidores, um fator marcante nas suas despesas mensais para desempenho de suas atividades. Assim, uma redução no valor desta energia representará um ganho financeiro para estes consumidores, melhorando a sua qualidade de vida no tocante à cidadania e inclusão social. Com relação à concessionária, há possibilidade de atendimento a novas cargas sem que haja investimentos para a ampliação de seu sistema de distribuição. O que, sem dúvida, 
Citação: Vasconcellos A. B., et al Análise da Relação Custo-Benefício de Projeto de Eficiência Energética no Setor Residencial. E\&S Engineering and Science, (2014), 1:1

representa o principal benefício adquirido pela concessionária neste tipo de projeto.

A substituição de refrigeradores e lâmpadas proporcionam uma Redução do Consumo Anual de energia elétrica de 1.748 MWh/ano e uma Redução de Demanda na Ponta de $792 \mathrm{~kW}$. A relação Custo-Benefício foi de 0,51 , valor inferior ao limite máximo estabelecido pela ANEEL, de 0,8 , para que projetos desta natureza possuam viabilidade econômica. Uma RCB 0,51 significa que para cada 51 centavos investidos no programa de eficiência energética, obtêm-se 1 real de benefício, o que torna o projeto viável economicamente e interessante tanto

\section{REFERÊNCIAS}

Mauro Araujo Almeida, Roberto Schaeffer, and Emilio Lèbre La Rovere, "The potential for electricity conservation and peak load reduction in the residential sector of Brazil," Energy, no. 26, pp. 413-429, 2001.

EnergyPlus, "Getting Started with EnergyPlus," University of Illinois and Ernest Orlando Lawrence Berkeley National Laboratory, 2013a.

Érika Fernanda Toledo Borges Leão, "Carta bioclimática de Cuiabá - Mato Grosso," Universidade Federal de Mato Grosso, 2007.

INMETRO, "Anexo V da Portaria INMETRO 50/2013," Instituto Nacional de Metrologia, Qualidade e Tecnologia, Rio de Janeiro, 2013.

Cleverson Sousa Camargo et al., "Simulação Computacional para Análise da Eficiência Energética e Conforto Térmico de uma Edificação Utilizando o Software EnergyPlus.," VIII CBPE - Congresso Brasileiro de Planejamento Energético 2012, 2012.

Energyplus, "Auxiliary EnergyPlus Programs," University of Illinois and Ernest Orlando Lawrence Berkeley National Laboratory, 2013b. para a concessionária, ao permitir a interligação de novos consumidores sem precisar ampliar o sistema elétrico, como para o consumidor, traduzindo-se na redução de sua fatura de energia elétrica.

Diante do exposto, pode-se dizer que ações conjuntas entre concessionária e consumidores, constituem atualmente o principal alicerce para o desenvolvimento de metodologias que permitem uma redução das perdas financeiras, técnicas, comerciais e sociais, impostas às distribuidoras e aos usuários, causadas pela utilização irracional dos recursos elétricos disponíveis e aparelhos não detentores do Selo PROCEL.

ANEEL, "Manual do Programa de Eficiência Energética," Agência Nacional de Energia Elétrica - ANEEL, Brasília, 2008b.

ANEEL, "Procedimentos do Programa de Eficiência Energética (PROPEE): Módulo 7 - Cálculo da Viabilidade," Agência Nacional de Energia Elétrica ANEEL, Brasília, 2013c.

ABRADEE, "Método para Determinação, Análise e Otimização das Perdas Técnicas em Sistemas de Distribuição. Relatório 19-34.," Associação Brasileira de Distribuidores de Energia Elétrica - ABRADEE, Rio de Janeiro, 1996.

ANEEL, "Resolução Homologatória $\mathrm{N}^{\circ} 1.506$, de 5 de Abril de 2013.," Agência Nacional de Energia Elétrica ANEEL, Brasília, 2013a.

Brasil. (2000) Presidência da República. Casa Civil. Dispõe sobre realização de investimentos em pesquisa e desenvolvimento e em eficiência energética por parte das empresas concessionárias, permissionárias e autorizadas do setor de energia elétrica, e dá outras providê. [Online]. http://www.planalto.gov.br/ccivil_03/leis/19991.html. 